\title{
LEBESGUE CONSTANTS FOR A FAMILY OF SUMMABILITY METHODS
}

\author{
KYUHEI IKENO
}

(Received June 15, 1965)

1. Introduction. The unboundedness of the sequence of Lebesgue constants implies the existence of a continuous function whose Fourier series diverges at a point, and this is also the case with many summability methods. The estimation of such constants for various summability methods has been calculated by K. Ishiguro [2], [3], A. E. Livingston [4], and L. Lorch [5], [6], [7], [8].

In this paper we shall study the behavior of the Lebesgue constants for a family of summability methods. A. Meir [9] has introduced a family of summability methods which is defined by two parameters $a$ and $q$, and has shown that this family contains Borel, Valiron, Euler, Taylor and $S_{\alpha^{-}}$ transformation.

If we define $L_{F}(a, q(p))$ by the Lebesgue constants for this family of summability methods, then we obtain the following formula:

$$
L_{F}(a, q(p))=\frac{2}{\pi^{2}} \log 4 a q(p)+A+O(\log q / \sqrt{q}) \text { as } p \rightarrow \infty,
$$

where $A$ is the constant such as

$$
A=-\frac{c}{\pi^{2}}+\frac{2}{\pi} \int_{0}^{1} \frac{\sin u}{u} d u-\frac{2}{\pi} \int_{1}^{\infty}\left(\frac{2}{\pi}-|\sin u|\right) \frac{d u}{u}
$$

and

$$
c=\int_{0}^{1} \frac{1-e^{-u}}{u}-\int_{1}^{\infty} \frac{1}{u e^{u}} d u
$$

which is Euler-Mascheroni's constant.

The proof of formula (1.1) consists of two parts; $1^{\circ}$ ) the case where $q=q(p)$ is integer and $\left.2^{\circ}\right)$ the case where $q=q(p)$ is not integer. In the last section we shall show that from (1.1) we can obtain Lebesgue constants for Borel, Valiron, Euler, Taylor and $S_{\alpha}$-transformation which are contained in this family of summability methods.

Finally I wish to express my gratitude to Professor G. Sunouchi for his kind suggestions. 
2. The Family $\mathbf{F}(\mathbf{a}, \mathbf{q}(\mathbf{p}))$ of Summability Methods. After A. Meir [9], let us say the summability matrix $\left[c_{p k}\right]$ belongs to $F(a, q(p))$ if it satisfies the following conditions : $p$ is a discrete or continuous parameter; $a$ is a positive constant; $q=q(p)$ is a positive increasing function which tends to infinity as $p \rightarrow \infty$; for every fixed $\delta: 1 / 2<\delta<2 / 3$

$$
c_{p k}=\sqrt{\frac{a}{\pi q}} e^{-\frac{a}{q}(k-q)^{2}}\left\{1+O\left(\frac{\mid k-q !+1}{q}\right)+O\left(\frac{|k-q|^{3}}{q^{2}}\right)\right\}
$$

as $p \rightarrow \infty$ uniformly in $k$ for $|k-q| \leqq q^{\delta}$,

$$
c_{p 0}+\sum_{|k-q|>q^{\delta}} k c_{p k}=O\left(\exp \left(-q^{\eta}\right)\right)
$$

where $\eta$ is some positive number independent of $p$, and

$$
c_{p k} \geqq 0 \text {. }
$$

It is known that the family $F(a, q(p))$ with appropriate $a$ and $q(p)$ contains such summability methods as Borel, Valiron, Euler, Taylor and $S_{\alpha^{-}}$ transformation, see G. H. Hardy [1] and A. Meir [9].

Let a function $f(x)$ be integrable in Lebesgue's sense over the interval $-\pi \leqq x \leqq \pi$ and periodic with period $2 \pi$.

If we define $S_{n}(f ; x)$ by the $n$-th partial sum of the Fourier series of $f(x)$ and $t_{p}(f ; x)$ by the transformation of $S_{n}(f ; x)$ by means of summability matrix $\left[c_{p k}\right]$, then we have

$$
t_{p}(f ; x)=\sum_{k=0}^{\infty} c_{p k} S_{k}(f ; x)=\sum_{k=0}^{\infty} \frac{1}{\pi} \int_{-\pi}^{\pi} f(x+u) \frac{c_{p k} \sin \left(k+\frac{1}{2}\right) u}{2 \sin u / 2} d u .
$$

When we suppose that for all $p$

$$
\begin{gathered}
\sum_{k=0}^{\infty} k\left|c_{p k}\right|<\infty, \\
t_{p}(f ; x)=\frac{1}{\pi} \int_{-\pi}^{\pi} f(x+u) \frac{1}{2 \sin u / 2} \sum_{k=0}^{\infty} c_{p k} \sin \left(k+\frac{1}{2}\right) u d u .
\end{gathered}
$$

If the summability matrix $\left[c_{p k}\right]$ belongs to $F(a, q(p))$, then the condition 
(2.4) is satisfied and Lebesgue constants for this methods $L_{F}(a, q(p))$ are defined as follows :

$$
L_{F}(a, q(p))=\frac{2}{\pi} \int_{0}^{\pi / 2}\left|\frac{1}{\sin u} \sum_{k=0}^{\infty} c_{p k} \sin (2 k+1) u\right| d u .
$$

3. Three Lemmas. To prove formula (1.1), we require the following three lemmas.

LEMMA 3.1. When $p$ tends to infinity, we get:

$$
\begin{gathered}
\frac{2}{\pi} \int_{0}^{\pi / 2} \frac{1}{\sin u} \sum_{|k-q| \leq q^{\delta}} \sqrt{\frac{a}{\pi q}} e^{-\frac{a}{q}(k-q)^{2}} \frac{|k-q|+1}{q}|\sin (2 k+1) u| d u \\
=O(\log q / \sqrt{q})
\end{gathered}
$$

and

$$
\begin{gathered}
\frac{2}{\pi} \int_{0}^{\pi / 2} \frac{1}{\sin u} \sum_{|k-q| \leqq q^{\delta}} \sqrt{\frac{a}{\pi q}} e^{-\frac{a}{q}(k-q)^{2}} \frac{|k-q|^{3}}{q^{2}}|\sin (2 k+1) u| d u \\
=O(\log q / \sqrt{q}) .
\end{gathered}
$$

ProOF. i) We can suppose $0<1 / q<\pi / 2$ for sufficiently large $p$, and set $I_{11}, I_{12}$ as follows:

$$
\begin{aligned}
& \frac{2}{\pi} \int_{0}^{\pi / 2} \frac{1}{\sin u} \sum_{|k-q| \leqq q^{\delta}} \sqrt{\frac{a}{\pi q}} e^{-\frac{a}{q}(k-q)^{2}} \frac{|k-q|+1}{q}|\sin (2 k+1) u| d u \\
& =\frac{2}{\pi}\left(\int_{0}^{1 / q}+\int_{1 / q}^{\pi / 2}\right) \frac{1}{\sin u} \sum_{|k-q| \leqq q^{\delta}} \sqrt{\frac{a}{\pi q}} e^{-\frac{a}{q}(k-q)^{2}} \frac{|k-q|+1}{q}|\sin (2 k+1) u| d u \\
& =I_{11}+I_{12} .
\end{aligned}
$$

Since we have

$$
\begin{aligned}
& \sum_{|k-q| \leqq q^{\delta}} \sqrt{\frac{a}{\pi q}} e^{-\frac{a}{q}(k-q)^{2}} \frac{|k-q|+1}{q}|\sin (2 k+1) u| \\
& \leqq \sqrt{\frac{a}{\pi q}} \sum_{|k-q| \leqq q^{\delta}} e^{-\frac{a}{q}(k-q)^{2}} \frac{|k-q|+1}{q}\{2|k-q|+(2 q+1)\}|u|
\end{aligned}
$$




$$
\begin{aligned}
& =O\left(\sqrt{\frac{a}{\pi q}} \sum_{|k-q| \leqq q^{\delta}} e^{-\frac{a}{q}(k-q)^{2}}\left(\frac{(k-q)^{2}}{q}+|k-q|+1\right)|u|\right) \\
& =O(\sqrt{q}|u|),
\end{aligned}
$$

then we obtain

$$
I_{11}=O\left(\sqrt{q} \int_{0}^{1 / q} \frac{u}{\sin u} d u\right)=O(1 / \sqrt{q}),
$$

and

$$
\begin{aligned}
I_{12} & =O\left(\int_{1 / q}^{\pi / 2} \frac{1}{\sin u} \sum_{|k-q| \leqq q^{\delta}} \sqrt{\frac{a}{\pi q}} e^{-\frac{a}{q}(k-q)^{2}} \frac{|k-q|+1}{q} d u\right) \\
& =O\left(\frac{1}{\sqrt{q}} \int_{1 / q}^{\pi / 2} \frac{d u}{\sin u}\right)=O(\log q / \sqrt{q}), \text { as } \quad p \rightarrow \infty .
\end{aligned}
$$

Therefore (3.1.1) has been proved.

ii) We can prove (3.1.2) by the same method as in (i).

We suppose that $0<1 / q<\pi / 2$ similar as in (i) and set $I_{21}, I_{22}$ as follows:

$$
\begin{aligned}
& \frac{2}{\pi} \int_{0}^{\pi / 2} \frac{1}{\sin u} \sum_{|k-q| \leqq q^{\delta}} \sqrt{\frac{a}{\pi q}} e^{-\frac{a}{q}(k-q)^{2}} \frac{|k-q|^{3}}{q^{2}}|\sin (2 k+1) u| d u \\
& \quad=\frac{2}{\pi}\left(\int_{0}^{1 / q}+\int_{1 / q}^{\pi / 2}\right) \frac{1}{\sin u} \sum_{|k-q| \leqq q^{\delta}} \sqrt{\frac{a}{\pi q}} e^{-\frac{\pi}{q}(k-q)^{2}} \frac{|k-q|^{3}}{q^{2}}|\sin (2 k+1) u| d u \\
& \quad=I_{21}+I_{22} .
\end{aligned}
$$

Since we have

$$
\begin{aligned}
\sum_{|k-q| \leqq q^{\delta}} & \sqrt{\frac{a}{\pi q}} e^{-\frac{a}{q}(k-q)^{2}} \frac{|k-q|^{3}}{q^{2}}|\sin (2 k+1) u| \\
& \leqq \sqrt{\frac{a}{\pi q}} \sum_{|k-q| \leqq q^{\delta}} e^{-\frac{a}{q}(k-q)^{2}} \frac{|k-q|^{3}}{q^{2}}\{2|k-q|+(2 q+1)\}|u| \\
= & O\left(\sqrt{\frac{a}{\pi q}} \sum_{|k-q| \leqq q^{\delta}} e^{-\frac{a}{q}(k-q)^{2}}\left(\frac{(k-q)^{4}}{q^{2}}+\frac{|k-q|^{3}}{q}\right)|u|\right) \\
= & O(\sqrt{q}|u|),
\end{aligned}
$$


then we obtain

$$
I_{21}=O\left(\sqrt{q} \int_{0}^{1 / q} \frac{u}{\sin u} d u\right)=O(1 / \sqrt{q})
$$

and

$$
\begin{aligned}
I_{22} & =O\left(\frac{2}{\pi} \int_{1 / q}^{\pi / 2} \frac{1}{\sin u} \sum_{|k-q| \leqslant q^{\delta}} \sqrt{\frac{a}{\pi q}} e^{-\frac{a}{q}(k-q)^{2}} \frac{|k-q|^{3}}{q^{2}} d u\right) \\
& =O\left(\frac{1}{\sqrt{q}} \int_{1 / q}^{\pi, 2} \frac{d u}{\sin u}\right)=O(\log q / \sqrt{q}) \text { as } p \rightarrow \infty
\end{aligned}
$$

Therefore (3.1.2) has been proved.

LEMMA 3.2. If $q=q(p)$ is an integer valued function of $p$, then we have

$$
\begin{aligned}
& \frac{2}{\pi} \int_{0}^{\pi / 2}\left|\frac{1}{\sin u} \sum_{|k-q| \leqslant q^{\delta}} \sqrt{\frac{a}{\pi q}} e^{-\frac{a}{q}(k-q)^{2}} \sin (2 k+1) u\right| d u \\
& \quad=\frac{2}{\pi} \int_{0}^{\pi / 2}\left|\frac{e^{-\frac{q u^{2}}{a}} \sin (2 q+1) u}{\sin u}\right| d u+o(1 / q), \text { as } \quad p \rightarrow \infty .
\end{aligned}
$$

ProOF. When we set $n=k-q(p)$, we have

$$
\begin{aligned}
& \sum_{|k-q| \leqq q^{\delta}} \sqrt{\frac{a}{\pi q}} e^{-\frac{a}{q}(k-q)^{2}} \sin (2 k+1) u \\
& =\mathfrak{S}\left\{e^{i(2 q+1) u} \sqrt{\frac{a}{\pi q}} \sum_{|n| \leqq q^{\delta}} e^{-\frac{a}{q} n^{2}+2 u n i}\right\} \\
& =\Im\left\{e^{i(2 q+1) u} \sqrt{\frac{a}{\pi q}}\left(\sum_{n=-\infty}^{+\infty}-\sum_{|n|>q^{\delta}}\right) e^{-\frac{q}{a} n^{2}+2 u n i}\right\} .
\end{aligned}
$$

Using the property of Theta function [10], we get

$$
\sqrt{\frac{a}{\pi q}} \sum_{n=-\infty}^{+\infty} e^{-\frac{a}{q} n^{2}+2 u n i}=\sum_{n=-\infty}^{+\infty} e^{-\frac{a}{q}(u-n \pi)^{2}}
$$

and for $0 \leqq u \leqq \pi / 2$ 


$$
\begin{aligned}
\sum_{|k-q| \leqq q^{\delta}} & \sqrt{\frac{a}{\pi q}} e^{-\frac{a}{q}(k-q)^{2}} \sin (2 k+1) u \\
= & \Im\left\{e^{i((2 q+1) u} \sum_{n=-\infty}^{+\infty} e^{-\frac{q}{a}(u-n \pi)^{2}}\right\}+O\left(\sum_{|n|>q^{\delta}} \sqrt{\frac{a}{\pi q}} e^{-\frac{a}{q} n^{2}}|\sin (2 n+2 q+1) u|\right) \\
= & e^{-\frac{q u u^{2}}{a}} \sin (2 q+1) u+O\left(q e^{-\frac{q u u^{2}}{4 a}}|u|\right)+O\left(q e^{-a q^{2 \delta-1}}|u|\right) \\
= & e^{-\frac{q u^{2}}{a}} \sin (2 q+1) u+O\left(q e^{-a q^{2 \delta-1}}|u|\right) .
\end{aligned}
$$

Therefore we get

$$
\begin{aligned}
& \frac{2}{\pi} \int_{0}^{\pi / 2}\left|\frac{1}{\sin u} \sum_{|n| \leqq q^{\delta}} \sqrt{\frac{a}{\pi q}} e^{-\frac{a n^{2}}{q}} \sin (2 n+2 q+1) u\right| d u \\
& \quad=\frac{2}{\pi} \int_{0}^{\pi / 2}\left|\frac{e^{-\frac{q u^{2}}{a}} \sin (2 q+1) u}{\sin u}\right| d u+o(1 / q) \text { as } p \rightarrow \infty .
\end{aligned}
$$

and consequently we have proved Lemma 3.2 .

LEMMA 3.3 Let $f(u, q)$ be defined over $q \geqq 0$ and $0 \leqq u \leqq \frac{\pi}{2}$, and let $\frac{\partial f}{\partial u}=f_{u}(u, q)$ exist for $q>0$, and $f_{u}(u, q)$ be integrable over $[0, \pi / 2]$.

$$
\text { If } \quad \int_{0}^{\pi / 2}\left|f_{u}(u, q)\right| d u=O(\sqrt{q}) \text { and } f\left(\frac{\pi}{2}, q\right)=O(\sqrt{q}) \text { as } q \rightarrow \infty,
$$

then we have

$$
\int_{0}^{\pi / 2} f(u, q)\left\{\frac{2}{\pi}-|\sin (2 q+1) u|\right\} d u=O(1 / \sqrt{q}), \quad \text { as } \quad q \rightarrow \infty
$$

In order to prove this lemma, see L. Lorch [5].

4. Lebesgue Constants. In this section we calculate Lebesgue constants for a family of summability methods whose matrix $\left[c_{p k}\right]$ belongs to $F(a, q(p))$.

THEOREM. Let $L_{F}(a, q(p))$ denote the Lebesgue constants for a family of summability methods whose matrix $\left[c_{p k}\right]$ belongs to $F(a, q(p))$.

Then we get the following formula:

(1. 1) $L_{F}(a, q(p))=\frac{2}{\pi^{2}} \log 4 a q(p)+A+O(\log q / \sqrt{q})$ as $p \rightarrow \infty$, 
where constant $A$ is defined by (1.2).

PROOF. $1^{\circ}$ ) The case where $q=q(p)$ is integer.

From (2.5) we have

$$
L_{F}(a, q(p))=\frac{2}{\pi} \int_{0}^{\pi / 2}\left|\frac{1}{\sin u}\left(\sum_{|k-q| \leqq q^{\delta}}+\sum_{|k-q|>\phi^{\delta}}\right) c_{p k} \sin (2 k+1) u\right| d u .
$$

We set $n, L(a, q)$ and $E$ as follows :

$$
\begin{gathered}
n=k-q(p) \\
L(a, q)=\frac{2}{\pi} \int_{0}^{\pi / 2}\left|\frac{1}{\sin u} \sum_{|k-q| \leqq \phi^{\delta}} c_{p k} \sin (2 k+1) u\right| d u \\
E=\frac{2}{\pi} \int_{0}^{\pi / 2}\left|\frac{1}{\sin u} \sum_{|k-q|>q^{\delta}} c_{p k} \sin (2 k+1) u\right| d u .
\end{gathered}
$$

Using (2.2), we have

$$
\begin{aligned}
|E| & =O\left(\int_{0}^{\pi / 2} \frac{1}{\sin u}\left(c_{p o}+\sum_{|k-q|>q^{\delta}} k c_{p k}\right)|u| d u\right) \\
& =O\left(e^{-q^{\eta}}\right)=o(1 / q)
\end{aligned}
$$

We get from Lemma 3.2,

$$
\begin{aligned}
& \frac{2}{\pi} \int_{0}^{\pi / 2}\left|\frac{1}{\sin u} \sum_{|n| \leqq q^{\delta}} e^{-\frac{a n^{2}}{q}} \sin (2 k+1) u\right| d u \\
& \quad=\frac{2}{\pi} \int_{0}^{\pi / 2}\left|\frac{1}{\sin u} e^{-\frac{q u^{2}}{a}} \sin (2 q+1) u\right| d u+o(1 / q) .
\end{aligned}
$$

Applying (4.3) and Lemma 3.1 to (4.1), we obtain

(4.5) $\quad L(a, q)=\frac{2}{\pi} \int_{0}^{\pi / 2} \mid \frac{1}{\sin u} \sum_{|n| \leqq q^{\delta}} \sqrt{\frac{a}{\pi q}} e^{-\frac{a_{n}{ }^{q}}{q}}$

$$
\left\{1+O\left(\frac{|n|+1}{q}\right)+O\left(\frac{|n|^{3}}{q^{2}}\right)\right\} \sin (2 k+1) u \mid d u
$$




$$
\begin{aligned}
& =\frac{2}{\pi} \int_{0}^{\pi / 2}\left|\frac{1}{\sin u} e^{-\frac{q u^{2}}{a}} \sin (2 q+1) u\right| d u+O(\log q / \sqrt{q}) \\
& =\frac{2}{\pi} \int_{0}^{\pi / 2}\left|\frac{e^{-\frac{q u^{2}}{a}}}{u} \sin (2 q+1) u\right| d u+O(1 / q)+O(\log q / \sqrt{q}) .
\end{aligned}
$$

If we define $L(q)$ as follows;

$$
L(q)=\frac{2}{\pi} \int_{0}^{\pi / 2} \frac{1}{u}|\sin (2 q+1) u| d u
$$

then from L. Lorch [5], [6] we obtain

$$
\begin{aligned}
L(q) & =\frac{4}{\pi^{2}} \log q-\frac{2}{\pi^{2}} \int_{0}^{\pi} \frac{\Gamma^{\prime}(u / \pi)}{\Gamma(u / \pi)} \sin u d u+O(1 / q) \\
& =\frac{4}{\pi^{2}} \log q+\frac{4}{\pi^{2}} \log \pi+\frac{2 c}{\pi^{2}}+A+O(1 / q),
\end{aligned}
$$

where $A$ and $c$ are defined by (1.2).

If we set $d(q)=L(q)-L(a, q)$, then from (4.5) we have

$$
\begin{aligned}
d(q) & =L(q)-L(a, q) \\
& =\frac{2}{\pi} \int_{0}^{\pi / 2} \frac{1}{u}\left(1-e^{-\frac{q u^{2}}{a}}\right)|\sin (2 q+1) u| d u \\
& =\frac{2}{\pi} \int_{0}^{\pi / 2} f(u, q)|\sin (2 q+1) u| d u+O(\log q / \sqrt{q})
\end{aligned}
$$

where $f(u, q)=\frac{1}{u}\left(1-e^{-\frac{q u^{2}}{a}}\right)$.

Since the function $f(u, q)$ satisfies the conditions of Lemma 3.3, (see L. Lorch [5]), we have

$$
\begin{aligned}
d(q) & =\frac{4}{\pi^{2}} \int_{0}^{\pi / 2} \frac{1}{u}\left(1-e^{-\frac{n u^{2}}{a}}\right) d u+O(\log q / \sqrt{q}) \\
& =\frac{2}{\pi^{2}} \int_{0}^{1} \frac{1}{u}\left(1-e^{-u}\right) d u+\frac{2}{\pi^{2}} \int_{1}^{q u^{2} / 4 a} \frac{1}{u}\left(1-e^{-u}\right) d u+O(\log q / \sqrt{q})
\end{aligned}
$$




$$
\begin{aligned}
& =\frac{2}{\pi^{2}} \log \frac{q \pi^{2}}{4 a}+\frac{2}{\pi^{2}}\left\{\int_{0}^{1} \frac{1}{u}\left(1-e^{-u}\right) d u-\int_{1}^{\infty} \frac{1}{u e^{u}} d u\right\} \\
& \quad+\frac{2}{\pi^{2}} \int_{q \pi^{2} / 4 a}^{\infty} \frac{d u}{u e^{u}}+O(\log q / \sqrt{q}) \\
& =\frac{2}{\pi^{2}} \log \frac{q \pi^{2}}{4 a}+\frac{2 c}{\pi^{2}}+O(\log q / \sqrt{q}) .
\end{aligned}
$$

Consequently we obtain

$$
\begin{aligned}
& L(a, q)=L(q)-d(q) \\
& =\frac{4}{\pi^{2}} \log q+\frac{4}{\pi^{2}} \log \pi+\frac{2 c}{\pi^{2}}+A-\frac{2}{\pi^{2}} \log \frac{q \pi^{2}}{4 a}-\frac{2 c}{\pi^{2}}+O(\log q / \sqrt{q}) \\
& =\frac{2}{\pi^{2}} \log 4 a q+A+O(\log q / \sqrt{q})
\end{aligned}
$$

From (4.1), (4.2), and (4.7), we get

$$
\begin{aligned}
L_{F}(a, q(p)) & =L(a, q)+o(1 / q) \\
& =\frac{2}{\pi^{2}} \log 4 a q(p)+A+O(\log q / \sqrt{q}), \text { as } p \rightarrow \infty .
\end{aligned}
$$

Therefore we have proved (1.1) when $q=q(p)$ is integer.

$2^{\circ}$ ) The case when $q=q(p)$ is not integer.

Let $[q]$ denote the integral part of $q=q(p)$ and $q_{0}=[q]+1$.

We set $D_{1}, D_{2}, D_{3}, D_{4}$ as follows :

$$
\begin{aligned}
& \left|\frac{2}{\pi} \int_{0}^{\pi / 2}\right| \frac{1}{\sin u} \sum_{|k-q| \leqq q^{\delta}} \sqrt{\frac{a}{\pi q}} e^{-\frac{a}{q}(k-q)^{2}} \sin (2 k+1) u \mid d u \\
& \quad-\frac{2}{\pi} \int_{0}^{\pi / 2}\left|\frac{1}{\sin u} \sum_{\left|k-q_{0}\right| \leqq q_{0}^{\delta}} \sqrt{\frac{a}{\pi q_{0}}} e^{-\frac{a}{q_{0}}\left(k-q_{0}\right)^{2}} \sin (2 k+1) u\right| d u \mid \\
& \leqq \frac{2}{\pi} \int_{0}^{\pi / 2} \frac{1}{\sin u}\left(\sum_{q<k \leqq q+q^{\delta}}+\sum_{q-q^{\delta} \leqq k<q}\right) \mid\left(\sqrt{\frac{a}{\pi q}} e^{-\frac{a}{q}(k-q)^{2}}\right. \\
& \left.-\sqrt{\frac{a}{\pi q_{0}}} e^{-\frac{a}{q_{0}}\left(k-q_{0}\right)^{2}}\right) \sin (2 k+1) u \mid d u
\end{aligned}
$$




$$
\begin{aligned}
& +\frac{2}{\pi} \int_{0}^{\pi / 2} \frac{1}{\sin u} \sum_{q+q^{\delta}<k \leqq q_{0}+q_{0}^{\delta}} \sqrt{\frac{a}{\pi q_{0}}} e^{-\frac{a}{q_{0}}\left(k-q_{0}\right)^{2}}|\sin (2 k+1) u| d u \\
& +\frac{2}{\pi} \int_{0}^{\pi / 2} \frac{1}{\sin u} \sum_{q-q^{\delta} \leqq k<q_{0}-q_{0}^{\delta}} \sqrt{\frac{a}{\pi q}} e^{-\frac{a}{q}\left(k-q^{2}\right)^{2}}|\sin (2 k+1) u| d u \\
& =D_{1}+D_{2}+D_{3}+D_{4},
\end{aligned}
$$

where we take $p$ large enough.

i) In case where $q<q_{0} \leqq k \leqq q+q^{\delta}$, we have

$$
0 \leqq\left(k-q_{0}\right) / \sqrt{q_{0}}<(k-q) / \sqrt{q}<(k-[q]) / \sqrt{[q]} .
$$

Hence the following inequality results:

$$
\begin{aligned}
&\left|\sqrt{\frac{a}{\pi q}} e^{-\frac{a}{q}(k-q)^{2}}-\sqrt{\frac{a}{\pi q_{0}}} e^{-\frac{a}{q_{0}}\left(k-q_{0}\right)^{2}}\right| \\
& \leqq \sqrt{\frac{a}{\pi q_{0}}}\left(e^{-\frac{a}{q_{0}}\left(k-q_{0}\right)^{2}}-e^{-\frac{a}{q}(k-q)^{2}}\right) \\
&=O\left(\frac{1}{\sqrt{q_{0}}} \int_{\left(k-q_{0}\right) / \sqrt{q_{0}}}^{(k-[q] / \mathcal{N} \overline{q]}}\left|x e^{-a x^{2}}\right| d x\right) \\
&=O\left(\frac{1}{\sqrt{q_{0}}} e^{-\frac{a}{q_{0}}\left(k-q_{0}\right)^{2}}\left(\frac{(k-[q])^{2}}{[q]}-\frac{\left(k-q_{0}\right)^{2}}{q_{0}}\right)\right) \\
&=O\left(\frac{1}{\sqrt{q_{0}}} e^{-\frac{a}{q_{0}}\left(k-q_{0}\right)^{2}}\left(\frac{\left(k-q_{0}\right)^{2}}{q_{0}^{2}}+\frac{\left|k-q_{0}\right|}{q_{0}}+\frac{1}{q_{0}}\right)\right) .
\end{aligned}
$$

We shall estimate $D_{1}$, by dividing the range of integration of $D_{1}$ in (4.8) into two parts. We set $D_{11}, D_{12}$ as follows:

$$
\begin{aligned}
D_{1}=\frac{2}{\pi}\left(\int_{0}^{1 / q}+\int_{1 / q}^{\pi / 2}\right) \frac{1}{\sin u} \sum_{q<k \leqq q+q^{\delta}} & \mid\left(\sqrt{\frac{a}{\pi q}} e^{-\frac{a}{q}(k-q)^{2}}\right. \\
& \left.-\sqrt{\frac{a}{\pi q_{0}}} e^{-\frac{a}{q_{0}}\left(k-q_{0}\right)^{2}}\right) \sin (2 k+1) u \mid d u \\
= & D_{11}+D_{12},
\end{aligned}
$$

where we can suppose $0<1 / q<\pi / 2$ for sufficiently large $p$. 
From (4.9), we get

$$
\begin{aligned}
D_{11}= & O\left(\int_{0}^{1 / q} \frac{1}{\sin u} \sum_{q<k \leqq q+q^{\delta}} \frac{1}{\sqrt{q_{0}}} e^{-\frac{a}{q_{0}}\left(k-q_{0}\right)^{2}}\right. \\
& \left.\times\left(\frac{\left(k-q_{0}\right)^{2}}{q_{0}{ }^{2}}+\frac{\left|k-q_{0}\right|}{q_{0}}+\frac{1}{q_{0}}\right)(2|k-q|+2 q+1) u d u\right) \\
= & O\left(\sqrt{q} \int_{0}^{1 / q} \frac{u}{\sin u} d u\right)=O(1 / \sqrt{q})
\end{aligned}
$$

and

$$
\begin{aligned}
D_{12} & =O\left(\int_{1 / q}^{\pi / 2} \frac{1}{\sin u} \sum_{q<k \leqq q+q^{\delta}} \frac{1}{\sqrt{q_{0}}} e^{-\frac{a}{q_{0}\left(k-q_{0}\right)^{2}}}\left(\frac{\left(k-q_{0}\right)^{2}}{q_{0}^{2}}+\frac{\left|k-q_{0}\right|}{q_{0}}+\frac{1}{q_{0}}\right) d u\right) \\
& =O\left(\frac{1}{\sqrt{q}} \int_{1 / q}^{\pi / 2} \frac{1}{\sin u} d u\right)=O(\log \dot{q} / \sqrt{q}) .
\end{aligned}
$$

'Therefore

$$
D_{1}=D_{11}+D_{12}=O(\log q / \sqrt{q}) .
$$

ii) In case where $k \leqq[q]<q<q_{0}$, we have

$$
\left(k-q_{0}\right) / \sqrt{q_{0}}<(k-q) / \sqrt{q}<(k-[q]) / \sqrt{[q]} \leqq 0 .
$$

Hence the following inequality results similarly to (4.9):

$$
\begin{aligned}
\left|\sqrt{\frac{a}{\pi q}} e^{-\frac{a}{q}(k-q)^{2}}-\sqrt{\frac{a}{\pi q_{0}}} e^{-\frac{a}{q_{0}}\left(k-q_{0}\right)^{2}}\right| \\
\quad \leqq \sqrt{\frac{a}{\pi q}}\left(e^{-\frac{a}{q}(k-q)^{2}}-e^{-\frac{a}{q_{0}}\left(k-q_{0}\right)^{2}}\right) \\
\quad=O\left(\frac{1}{\sqrt{q}} \int_{\left(k-q_{0}\right) / \sqrt{q_{0}}}^{(k-[q]) / \sqrt{[q]}} \mid d x\right) \\
=O\left(\frac{1}{\sqrt{[q]}} e^{-\frac{a}{[q]}(k-[q])^{2}}\left(\frac{\left(k-q_{0}\right)^{2}}{q_{0}}-\frac{(k-[q])^{2}}{[q]}\right)\right) \\
=O\left(\frac{1}{\sqrt{[q]}} e^{-\frac{a}{[q]}(k-[q])^{2}}\left(\frac{(k-[q])^{2}}{[q]^{2}}+\frac{|k-[q]|}{[q]}+\frac{1}{[q]}\right)\right) .
\end{aligned}
$$


We divide the range of integration of $D_{2}$ in (4.8) into two parts for sufficiently large $p$ and set $D_{21}, D_{22}$ as follows :

$$
\begin{aligned}
D_{2}=\frac{2}{\pi}\left(\int_{0}^{1 / q}+\int_{1 / q}^{\pi / 2}\right) \frac{1}{\sin u} \sum_{q-q^{\delta} \leqq k<q} \mid\left(\sqrt{\frac{a}{\pi q}} e^{-\frac{a}{q}(k-q)^{2}}\right. \\
\left.\quad-\sqrt{\frac{a}{\pi q_{0}}} e^{-\frac{a}{q_{0}}\left(k-q_{0}\right)^{2}}\right) \sin (2 k+1) u \mid d u \\
=D_{21}+D_{22} .
\end{aligned}
$$

From (4.11), we get

$$
\begin{aligned}
D_{21}= & O\left(\int^{1 / q} \frac{1}{\sin u} \sum_{q-q^{\delta} \leqq k<q} \frac{1}{\sqrt{[q]}} e^{-\frac{a}{[q]}(k-[q])^{2}}\right. \\
& \left.\times\left(\frac{(k-[q])^{2}}{[q]^{2}}+\frac{|k-[q]|}{[q]}+\frac{1}{[q]}\right)(2|k-q|+2 q+1) u d u\right) \\
= & O\left(\sqrt{q} \int_{0}^{1 / q} \frac{u}{\sin u} d u\right)=O(1 / \sqrt{q})
\end{aligned}
$$

and

$$
\begin{aligned}
& D_{22}=O\left(\int_{1 / q}^{\pi / 2} \frac{1}{\sin u} \sum_{q-q^{\delta} \leqq k<q} \frac{1}{\sqrt{[q]}} e^{-\frac{a}{[q]}(k-\{q])^{2}}\left(\frac{(k-[q])^{2}}{[q]^{2}}+\frac{|k-[q]|}{[q]}+\frac{1}{[q]}\right) d u\right) \\
& =O\left(\frac{1}{\sqrt{q}} \int_{1 / q}^{\pi / 2} \frac{1}{\sin u} d u\right)=O(\log q / \sqrt{q}) .
\end{aligned}
$$

Therefore

$$
D_{2}=D_{21}+D_{22}=O(\log q / \sqrt{q}) \text {. }
$$

Next we shall estimate $D_{3}, D_{4}$ and we get for sufficiently large $p$,

$$
\begin{aligned}
D_{3} & =\frac{2}{\pi} \int_{0}^{\pi / 2} \frac{1}{\sin u} \sum_{q+q^{\delta}<k \leqq q_{0}+q_{0} \delta} \sqrt{\frac{a}{\pi q_{0}}} e^{-\frac{a}{q_{0}}\left(k-q_{0}\right)^{2}}|\sin (2 k+1) u| d u \\
& =O\left(\sqrt{q} e^{-a q^{2 \delta-1}} \int_{0}^{\pi / 2} \frac{u}{\sin u} d u\right)=O\left(\sqrt{q} e^{-a_{q} \delta \delta-1}\right) \\
& =o(1 / q),
\end{aligned}
$$


and

(4. 14) $\quad D_{4}=\frac{2}{\pi} \int_{0}^{\pi / 2} \frac{1}{\sin u} \sum_{q-q^{\delta} \leqq k<q_{0}-q_{0} \delta} \sqrt{\frac{a}{\pi q}} e^{-\frac{a}{q}(k-q)^{2}}|\sin (2 k+1) u| d u$

$$
\begin{aligned}
& =O\left(\sqrt{q} e^{-a q^{2 \delta-1}} \int_{0}^{\pi / 2} \frac{u}{\sin u} d u\right)=O\left(\sqrt{q} e^{-a q^{2 \delta-1}}\right) \\
& =o(1 / q) .
\end{aligned}
$$

Using (4.8), (4.10), (4.12), (4.13), (4.14) and setting $n_{0}=k-q_{0}$, we obtain

$$
\begin{aligned}
& \frac{2}{\pi} \int_{0}^{\pi / 2}\left|\frac{1}{\sin u} \sum_{|k-q| \leqq q^{\delta}} \sqrt{\frac{a}{\pi q}} e^{-\frac{a}{q}(k-q)^{2}} \sin (2 k+1) u\right| d u \\
& \quad=\frac{2}{\pi} \int_{0}^{\pi / 2}\left|\frac{1}{\sin u} \sum_{\left|n_{0}\right| \leqq q_{0} \delta} \sqrt{\frac{a}{\pi q_{0}}} e^{-\frac{a n_{0} 0^{2}}{q_{0}}} \sin (2 k+1) u\right| d u+O(\log q / \sqrt{q}) .
\end{aligned}
$$

Since we have from (4.7)

$$
\begin{gathered}
\frac{2}{\pi} \int_{0}^{\pi / 2}\left|\frac{1}{\sin u} \sum_{\left|n_{0}\right| \leqq q_{0} \delta} \sqrt{\frac{a}{\pi q_{0}}} e^{-\frac{a n_{0}{ }^{2}}{q_{0}}} \sin (2 k+1) u\right| d u \\
=\frac{2}{\pi^{2}} \log 4 a q_{0}+A+O\left(\log q_{0} / \sqrt{q_{0}}\right)
\end{gathered}
$$

we obtain

$$
\begin{gathered}
\frac{2}{\pi} \int_{0}^{\pi / 2}\left|\frac{1}{\sin u} \sum_{|k-q| \leqq q^{\delta}} \sqrt{\frac{a}{\pi q}} e^{-\frac{a}{q}(k-q)^{2}} \sin (2 k+1) u\right| d u \\
=\frac{2}{\pi^{2}} \log 4 a q(p)+A+O(\log q / \sqrt{q}) .
\end{gathered}
$$

From Lemma 3.1, (4.3) and (4.15), we have

$$
\begin{aligned}
L_{F}(a, q(p)) & =\frac{2}{\pi} \int_{0}^{\pi / 2}\left|\frac{1}{\sin u} \sum_{|k-q| \leqq q^{\delta}} \sqrt{\frac{a}{\pi q}} e^{-\frac{a}{q}(k-q)^{2}} \sin (2 k+1) u\right| d u+O(\log q / \sqrt{q}) \\
& =\frac{2}{\pi^{2}} \log 4 a q(p)+A+O(\log q / \sqrt{q}), \text { as } p \rightarrow \infty .
\end{aligned}
$$


Thus we have obtained the Lebesgue constants for a family of summability methods whose matrix $\left[c_{p k}\right]$ belongs to $F(a, q(p))$.

\section{Lebesgue constants for Borel, Valiron, Euler, Taylor and $\boldsymbol{S}_{\alpha^{-}}$ transformation.}

i) Borel-transformation. See L. Lorch [5].

The summability matrix of this transformation is defined by

$$
c_{p k}=e^{-p} \frac{p^{k}}{k !} \quad(k=0,1,2, \cdots),
$$

where $p>0, a=\frac{1}{2}$, and $q=p$, see A. Meir [9].

Therefore from (1.1) we get Lebesgue constants for Borel-transformation $L_{B}$ as follows :

$$
L_{B}=L_{F}\left(\frac{1}{2}, p\right)=\frac{2}{\pi^{2}} \log 2 p+A+O(\log p / \sqrt{p})
$$

This Lebesgue constants have been obtained already by L. Lorch [5] whose remainder term is $O(1 / \sqrt{p})$.

ii) Valiron-transformation.

The summability matrix is defined by

$$
c_{p k}=\sqrt{\frac{\alpha}{\pi p}} e^{-\frac{\alpha}{p}(k-p)^{2}} \quad(p=1,2, \cdots, \quad k=0,1,2, \cdots)
$$

where $\alpha>0, a=\alpha$ and $q=p$, see A. Meir [9].

Therefore from Theorem $1^{\circ}$ ) in section 4 we get Lebesgue constants for Valiron-transformation $L_{(v, \alpha)}$ as follows :

$$
L_{(V, \alpha)}=L_{F}(\alpha, p)=\frac{2}{\pi^{2}} \log 4 \alpha p+A+O(1 / \sqrt{p})
$$

iii) Euler-transformation. See L. Lorch [7] and A. E. Livingston [4]. The summability matrix of this transformation is defined by

$$
c_{p k}=\left(\begin{array}{c}
p \\
k
\end{array}\right) \alpha^{k}(1-\alpha)^{p-k}, \quad(p=1,2, \cdots, k=0,1,2, \cdots)
$$

where $0<\alpha<1, a=1 / 2(1-\alpha)$ and $q=\alpha p$, see A .Meir [9]. 
Therefore we get from (1.1) Lebesgue constants for Euler-transformation $L_{(E, \alpha)}$ as follows :

$$
\begin{aligned}
L_{(E, \alpha)} & =L_{F}(1 / 2(1-\alpha), \alpha p) \\
& =\frac{2}{\pi^{2}} \log \frac{2 \alpha p}{1-\alpha}+A+O(\log p / \sqrt{p}) .
\end{aligned}
$$

L. Lorch has obtained Lebesgue constants for $\left(E, \frac{1}{2}\right)$ in [7] and has shown that the remainder term is $O(1 / \sqrt{p})$.

iv) Taylor-transformation. See K. Ishiguro [2].

The summability matrix is defined by

$$
c_{p k}= \begin{cases}0 & (0 \leqq k \leqq p-1) \\
r^{p+1}\left(\begin{array}{l}
k \\
p
\end{array}\right)(1-r)^{k-p} & (p \leqq k)\end{cases}
$$

where $0<r<1, a=r / 2(1-r)$ and $q=p / r$, see A. Meir [9].

Therefore we get from (1.1) Lebesgue constants for Taylor-transformation $L_{(T, r)}$ as follows :

$$
\begin{aligned}
L_{(T, r)} & =L_{F}(r / 2(1-r), p / r) \\
& =\frac{2}{\pi^{2}} \log \frac{2 p}{1-r}+A+O(\log p / \sqrt{p}) .
\end{aligned}
$$

v) $S_{\alpha}$-transformation. See K. Ishiguro [3].

The summability matrix of this transformation is defined by

$$
c_{p k}=(1-\alpha)^{p+1}\left(\begin{array}{c}
p+k \\
k
\end{array}\right) \alpha^{k} \quad(k=0,1,2, \cdots, p=1,2, \cdots)
$$

where $0<\alpha<1, a=(1-\alpha) / 2$ and $q=\alpha p /(1-\alpha)$, see A. Meir [9].

Therefore we get from (1.1) Lebesgue constants for $S_{\alpha}$-transformation $L_{(s, \alpha)}$ as follows :

$$
\begin{aligned}
L_{(s, \alpha)} & =L_{F}((1-\alpha) / 2, \alpha p /(1-\alpha)) \\
& =\frac{2}{\pi^{2}} \log 2 \alpha p+A+O(\log p / \sqrt{p}) .
\end{aligned}
$$




\section{REFERENCES}

[1] G. H. Hardy, Divergent Series, Oxford Press, 1949.

[2] K. IsHiguro, The Lebesgue Constants for $(\boldsymbol{\gamma}, r)$ Summation of Fourier Series, Proc. Japan Acad., 36, 8(1960), 470-474.

[3] K. IshiguRo, Über das $S_{\alpha}$-Verfahren bei Fourier-Reihen, Math. Zeitschr., 80(1962), 4-11.

[4] A. E. Livingston, The Lebesgue Constants for Euler $(E, p)$ Summation of Fourier Series, Duke Math. Journ., 21(1954), 309-313.

[5] L. LoRCH, The Lebesgue Constants for Borel Summability, Duke Math. Journ., 11 (1944), 459-467.

[6] L. LORCH, On Fejér's Calculation of the Lebesgue Constants, Bulletin of the Calcutta Math. Soc., 37(1945), 5-8.

[7] L. LORCH, The Lebesgue Constants for $(E, 1)$ Summation of Fourier Series, Duke Math. Journ., 19(1952), 45-50.

[8] L. LORCH AND D. J. NEWMAN, The Lebesgue Constants for regular Hausdorff Methods, Canadian Journ. of Math., 13(1961), 283-298.

[9] A. MEIR, Tauberian Constants for a family of transformations, Annals of Math., 78 (1963), 594-599.

[10] E. T. Whittaker AND G. N. Watson, A course of Modern Analysis, Cambridge University Press, 1935.

\section{AKITA UNIVERSITY.}

